\title{
Temperature distribution in a composite rod, taking into account nonlocal spatial effects
}

\author{
George Kuvyrkin, Inga Savelyeva, and Daria Kuvshinnikova*
}

Department of Applied Mathematics, Bauman Moscow State Technical University, 105005, 2nd Baumanskaya st 5, Moscow, Russian Federation

\begin{abstract}
One of the important directions in the field of creating new structural and functional materials is the study of materials with a complex structure and unique strength or temperature properties. Due to the heterogeneity of their structure, such materials are called "structurally sensitive". The heterogeneity of the structure of such materials is justified by the technological features of their creation. Structurally sensitive materials can be obtained by compacting nanopowders, deposition on a substrate, etc. The paper considers a mathematical model of heat propagation in a composite rod. At one of the ends of the rod, a protective coating of structurally sensitive material is used. Using the finite element method, numerical solutions are found for a problem with conditions of ideal thermal contact of rod parts. The results of the numerical calculation for various materials are given. The influence of material parameters on the heat distribution in the rod is analysed.
\end{abstract}

\section{Nomenclature}

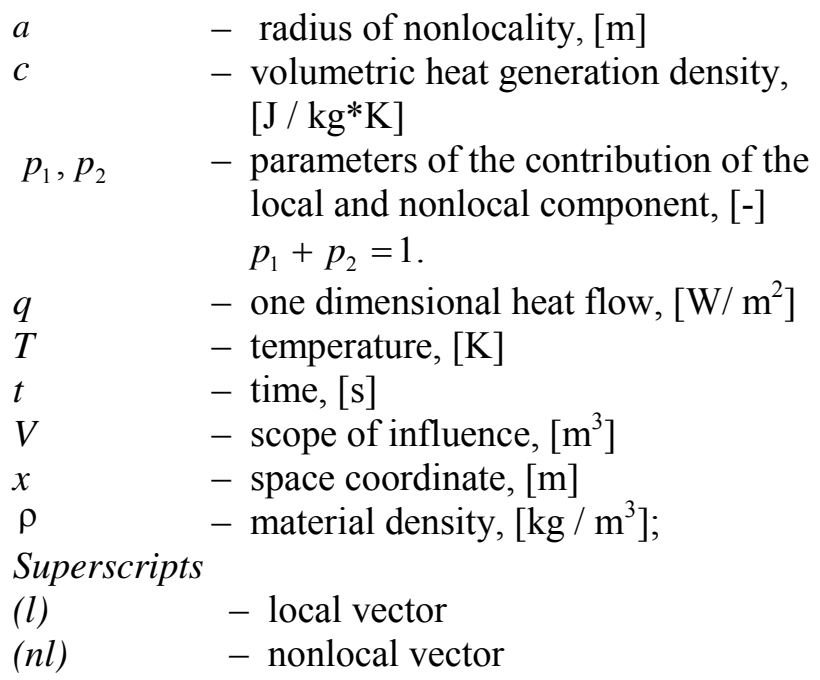

\section{Introduction}

The need for nonlocal thermodynamic models in science and technology emerged in the last century when scientists tried to explain the effects obtained in the experiments of Kapitsa (Kapitsa's phonon scattering). These effects could not be explained within the framework of the Fourier diffusion theory. Similar phenomena are observed in the molecular-dynamic modeling of heat transfer in nanowires. Experiments show the presence of thermostated sources that change the conductivity property of materials $[1-3]$. Such studies were further developed using mathematical tools for constructing thermodynamic theories that take into account nonlocal effects. The presence of spatial nonlocal effects observed within the framework of heat transfer was introduced using integral models including local temperature field gradients, an integral relationship between the temperature gradient and the function of attenuation of the effect of the temperature gradient. Nonlocal interpretation available suggested by Eringen. The development of this theory to describe the nonlocal behavior of materials under conditions of high-intensity surface illumination is considered in $[4,5]$.

Of interest is the case when one part of the rod is made of a homogeneous material, without a structural feature, and the other is of a material obtained by modern nanotechnology. In this case, the model can be applied to simulate a rod with a protective coating of a structure-sensitive material.

\section{Mathematical model}

Let the rod consist of two parts with different densities $\rho_{1}$ and $\rho_{2}$, heat capacity coefficients $c_{1}$ and $c_{2}$, and thermal conductivity $\lambda_{1}$ and $\lambda_{2}$, and the left part of the rod consists of structurally sensitive material.

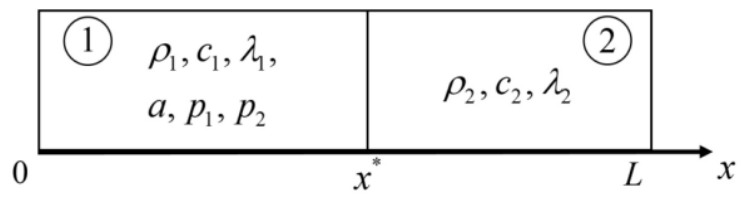

Fig. 1.The simplified composite rod model 
We write the heat conduction equations for the left and right parts of the rod, respectively:

$$
\begin{array}{ll}
\rho_{1} c_{1} \frac{\partial T_{1}(x, t)}{\partial t}=-\frac{\partial q^{(n l)}(x, t)}{\partial x}, & x \in\left(0, x^{*}\right), t>0, \\
\rho_{2} c_{2} \frac{\partial T_{2}(x, t)}{\partial t}=-\frac{\partial q^{(l)}(x, t)}{\partial x}, & x \in\left(x^{*}, L\right), t>0 .
\end{array}
$$

The Fourier thermal conductivity law for an isotropic homogeneous body:

$$
q^{(l)}(x, t)=-\lambda_{2} \frac{\partial T_{2}(x, t)}{\partial x} .
$$

The effect of nonlocality of the environment is that the physical characteristics of the elements of the structure-sensitive material are influenced by other surrounding elements of the structure. In this regard, the expression for the heat flux can be written in the form [4]

$$
q^{(n l)}(x, t)=-p_{1} \lambda_{1} \frac{\partial T_{1}(x, t)}{\partial x}-p_{2} \lambda_{1} \int_{V} \varphi\left(\left|x^{\prime}-x\right|\right) \frac{\partial T_{1}\left(x^{\prime}, t\right)}{\partial x^{\prime}} d x^{\prime},
$$

where $\varphi\left(\left|x^{\prime}-x\right|\right)$ - the function of the influence of nonlocality, and:

$$
\int_{V} \varphi\left(\left|x^{\prime}-x\right|\right) d x^{\prime}=1
$$

The function of the influence of nonlocality in this paper will be in the form [4]:

$$
\varphi\left(\left|x^{\prime}-x\right|\right)=\frac{1}{2 a} e^{-\frac{\left|x^{\prime}-x\right|}{a}}, \quad\left|x^{\prime}-x\right|<a .
$$

The parameters $p_{1}$ and $p_{2}$ are responsible for the contribution of the local and nonlocal component in the expression for the heat flux, and $p_{1}+p_{2}=1$.

Similar relationships are used in the mechanics of a deformable solid to find the stress distribution taking into account the effect of nonlocality of the medium [6].

The initial conditions for the rod are:

$$
T_{1}(x, 0)=T_{0}=\text { const }, \quad T_{2}(x, 0)=T_{0}=\text { const },
$$

where $T_{0}$ is the initial temperature of the rod.

The boundary conditions of the second kind:

$$
\begin{gathered}
-\lambda_{1} p_{1} \frac{\partial T_{1}(0, t)}{\partial x}-\lambda_{1} p_{2} \int_{V} \varphi\left(\left|x^{\prime}\right|\right) \frac{\partial T_{1}\left(x^{\prime}, t\right)}{\partial x^{\prime}} d x^{\prime}=q_{1}(t), \\
\lambda_{2} \frac{\partial T_{2}(L, t)}{\partial x}=q_{2}(t)
\end{gathered}
$$

where $q_{1}(t), q_{2}(t)$ are heat flows on the left and right ends of the rod, respectively.

At the junction of the two parts $x^{*}$ of the rod, there is an ideal thermal contact:

$$
T_{1}\left(x^{*}, t\right)=T_{2}\left(x^{*}, t\right),
$$

$$
\begin{aligned}
p_{1} \lambda_{1} \frac{\partial T_{1}\left(x^{*}, t\right)}{\partial x}+ & p_{2} \lambda_{1} \int_{x^{*}-a}^{x^{*}} \varphi\left(\mid x^{\prime}-x *\right) \frac{\partial T_{1}\left(x^{\prime}, t\right)}{\partial x^{\prime}} d x^{\prime}= \\
& =\lambda_{2} \frac{\partial T_{2}\left(x^{*}, t\right)}{\partial x} .
\end{aligned}
$$

\section{Results}

Consider a model in which the left part of the rod is made of titanium nitride and the main part of the steel.

Characteristics of materials [7 - 9]:

$T i N: \rho_{1}=5400 \mathrm{~kg} / \mathrm{m}^{3}, c_{1}=600 \mathrm{~J} /(\mathrm{kg} * K)$,

$$
\lambda_{1}=41.8 \mathrm{~W} /(\mathrm{m} * \mathrm{~K}) \text {. }
$$

Steel : $\rho_{2}=7800 \mathrm{~kg} / \mathrm{m}^{3}, c_{2}=460 \mathrm{~J} /(\mathrm{kg} * K)$,

$$
\lambda_{2}=22.4 \mathrm{~W} /(\mathrm{m} * K) \text {. }
$$

Copper: $\rho_{2}=8940 \mathrm{~kg} / \mathrm{m}^{3}, c_{2}=485 \mathrm{~J} /(\mathrm{kg} * K)$,

$$
\lambda_{2}=401 \mathrm{~W} /(\mathrm{m} * K) \text {. }
$$

Let us set $q_{1}=10^{5} \mathrm{~W} / \mathrm{m}^{2}$ on the left boundary, and zero heat flow on the right. Initial temperature $T_{0}=400 \mathrm{~K}$. Figures $2-3$ show the temperature distribution at time points $t=10^{3} \mathrm{~s}$ and $t=10^{4} \mathrm{~s}$, respectively. We see that as the contribution parameter of the local component increases, the value of the temperature at the boundary decreases. The radius of nonlocality $a$ in this model is dependent on the space step $h$ (in numerical simulation $h=10^{-7} \mathrm{~m}$ ). It should be noted that with an increase in the nonlocality radius, the temperature at the boundary increases, which indicates the need to take into account nonlocal effects when constructing a model. It is also worth noting that over time the boundary of the temperature between the parts of the rod becomes more significant and the effect of the coating of structurally sensitive material on the temperature distribution can be traced.

Next, we consider a model in which the left part of the rod is made of titanium nitride, and the main part is made of copper $[10,11]$.

Figures $4-5$ show the temperature distribution at time points $t=10^{3} \mathrm{~s}$ and $t=10^{4} \mathrm{~s}$, respectively. The boundary and initial conditions are chosen similarly to the case of a titanium nitride rod and steel.

Studies, as in the cases presented in Figures $4-5$, are presented depending on the contribution parameter of the local component $p_{1}$ and the diameter of the nonlocality $a$. From the results presented in Figures $4-5$, it can be seen that when using a coating of structurally sensitive material, it is possible to protect the base material from excessive heating, since, due to the properties of such a material, the temperature of the core of the rod decreases significantly. 


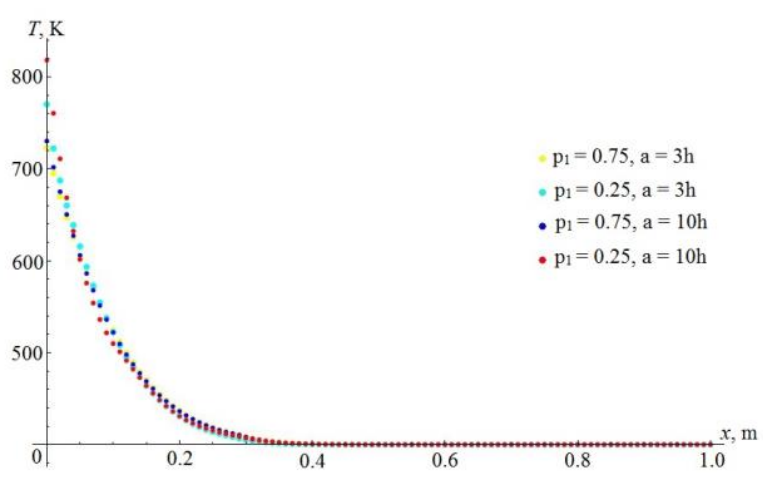

Fig. 2. The temperature distribution in the composite rod of titanium nitride and steel at the time $t=10^{3} \mathrm{~s}$

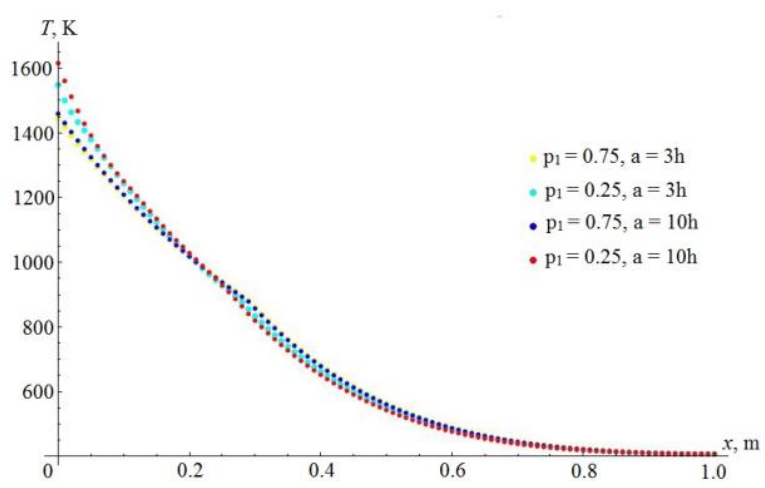

Fig. 3. The temperature distribution in the composite rod of titanium nitride and steel at the time $t=10^{4} \mathrm{~s}$

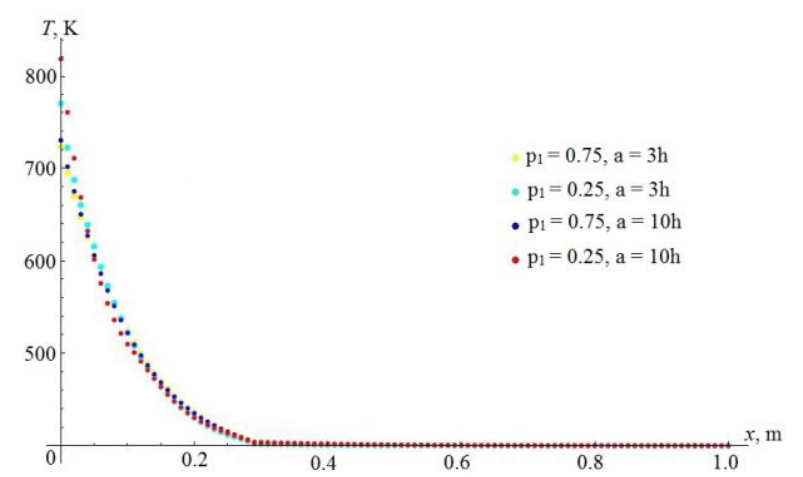

Fig. 4. The temperature distribution in the composite rod of titanium nitride and copper at the time $t=10^{3} \mathrm{~s}$

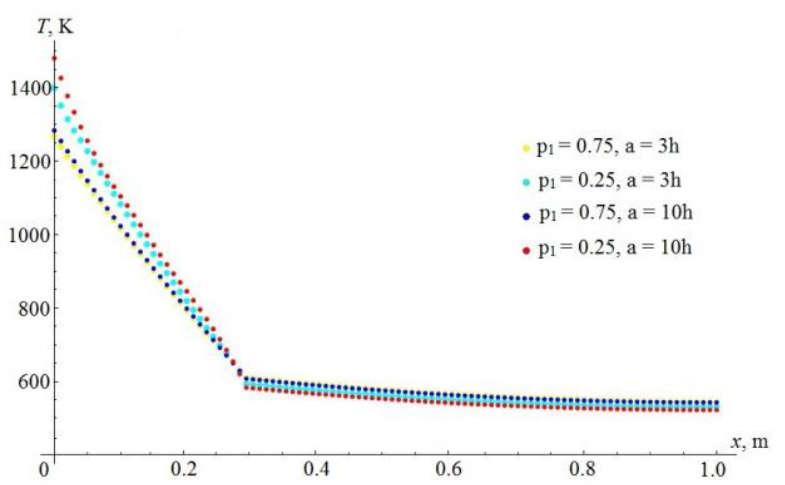

Fig. 5. The temperature distribution in the composite rod of titanium nitride and copper at the time $t=10^{4} \mathrm{~s}$

\section{Conclusions}

The solution to the problem of the propagation of heat in a rod with a protective coating of a structure-sensitive material is obtained. It is shown that the use in one part of a composite rod of structurally sensitive material with the presence of nonlocal effects in space provides the necessary level of protection of the second part of the rod against heat. This result shows that the resulting model of heat propagation can be used for the further development of models of structural materials.

The research was supported by the Russian Foundation for Basic Research projects No. 18-38-20108.

\section{References}

1. R.A. Andrievskiy, Zhurnal Ross. Khim. Ob-va im. D.I.Mendeleeva, Nanomaterials: the concept and modern problems, 46(5), pp. 50-56 (2002)

2. R.A. Andrievskiy, A.M. Glezer, Phys.Usp., Strength of nanostructures, 52(4), pp. 315-334 (2009)

3. E. Roduner, J. Chem Soc Rev, Size matters: why nanomaterials are different, 35(5), pp. 83-92 (2006)

4. G.N. Kuvyrkin, I.Y. Savelyeva, D.A. Kuvshynnikova, Journal of Physics: Conference Series, Mathematical model of the heat transfer process taking into account the consequences of nonlocality in structurally sensitive materials, 991(1) (2018)

5. V.S. Zarubin, G.N. Kuvyrkin, Mathematical Models of Continuum Mechanics and Electrodynamics (IMGTU im. Baumana, Moscow, 2008)

6. A.C. Eringen, Nonlocal Continuum Field Theories (New - York-Berlin - Heidelberg: Springer - Verlag, 2002)

7. G.N. Kuvyrkin, I.Yu. Svelyeva, A.V. Zhuravskii, Journal of Engineering Physics and Thermophysics, Mathematical Modeling of Chemical Vapor Deposition of Material on a Curvilinear Surface, 89(6), pp. 1-6 (2016)

8. V.S. Zarubin, G.N. Kuvyrkin, I.Yu. Savel'eva, High Temperature, Critical and Optimal Thicknesses of Thermal Insulation in Radiative-Convective Heat Transfer, 54(6), pp. 831-836 (2016)

9. V.S. Zarubin, G.N. Kuvyrkin, I.Y. Savelyeva, High Temperature, Radiative-conductive heat transfer in a spherical cavity, 53(2), pp. 234-239 (2015)

10. I.Yu. Savelyeva, Mechanics of Solids, Influence of medium nonlocality on distribution of temperature and stresses in elastic body under pulsed heating, 53(3), pp. 277-283 (2018)

11. G.N. Kuvyrkin, I.Y. Savelyeva, A.V. Zhuravsky, Mathematical Models and Computer Simulations, Numerical Simulation of Vapor-Phase Epitaxy with Allowance for Diffusion Processes, 10(3), pp. 299307 (2018) 